\title{
NOTA SOBRE LA PRESENCIA DE LA DIATOMEA ASTEROMPHALUS CLEVEANUS GRUNOW EN AGUAS DE BAJA CALIFORNIA Y SU RELACION CON A. FLABELLATUS (BREB.) GREV.
}

\author{
David U. Hernandez-Becerril \\ Centro de Investigaciones de Quintana Roo \\ Depto. de Ecología Acuática \\ Apdo. Postal 424 \\ 77000 Chetumal, Q. R., México
}

\begin{abstract}
RESUMEN
Se registra la presencia de la diatomea $A$. cleveanus en aguas de Baja California, detectada en muestras de red de esa región. Esta especie es descrita tomando en cuenta observaciones de microscopio de luz. La morfología es muy similar a la de $A$. flabellatus, pero se anotan las diferencias más claras entre ambas. También se discute su distribución, considerándose como una especie tropical y subtropical, nerítica y litoral, poco frecuente.
\end{abstract}

\begin{abstract}
The diatom A. cleveanus is recorded from waters of Baja California; it was encountered in net samples of that area. This species is described from light microscopy observations. Its morphology is very similar to that of $A$. flabellatus, but the main differences between the two species are pointed out. The distribution of $A$. cleveanus is discussed, and it is considered to be a tropical and subtropical, neritic and littoral, not frequent species.
\end{abstract}

\section{INTRODUCCION}

El análisis de material fitoplanctónico de red colectado en el Golfo de California y costas de Baja California ha brindado la oportunidad de encontrar nuevos registros de dinoflagelados (Hernández-Becerril, 1988a, 1988b) y de diatomeas (en preparación) en el Golfo de California. En particular, el estudio de algunas especies de la diatomea del género Asteromphalus aportó datos nuevos sobre su morfología y taxonomía (Hernández-Becerril, $1989,1991)$. Dentro de este género también se detectó el nuevo registro en el Golfo de California de $\boldsymbol{A}$. ingens Simonsen, especie originalmente descrita en el Océano Indico (Simonsen, 1974) y recientemente confundida con $A$. robustus Castracane en esa área (Atilano Silva, 1987).

Este trabajo señala el hallazgo de Asteromphalus cleveanus Grunow en la costa oeste de Baja California. Esta especie tiene gran similitud con A. flabellatus (Bréb.) Grev., ampliamente distribuida en aguas tropicales y subtropicales, lo que ocasiona dificultad en distinguirlas y posibles confusiones en su identificación. 


\section{MATERIAL Y METODOS}

El material estudiado corresponde a muestras de red colectadas en varios puntos dentro del Golfo de California y costas de Baja California (Fig. 1). El material preservado fue enjuagado para eliminar sales y fijadores; para el estudio de las diatomeas, el material enjuagado fue tratado con oxidantes $\left(\mathrm{KMnO}_{4}\right)$ y ácido $(\mathrm{HCl})$ para observar valvas sin contenido orgánico (Hasle, 1978). Se elaboraron preparaciones permanentes tanto de material enjuagado como libre de contenido orgánico, para su observación al microscopio de luz $(\mathrm{ML})$, usando un Olympus Bh con cámara integrada. El material tratado se utilizó para hacer observaciones en microscopio electrónico de transmisión (MET) JEOL 1200 EX, colocando una pequeña porción de material con ayuda de una micropipeta sobre rejillas previa-

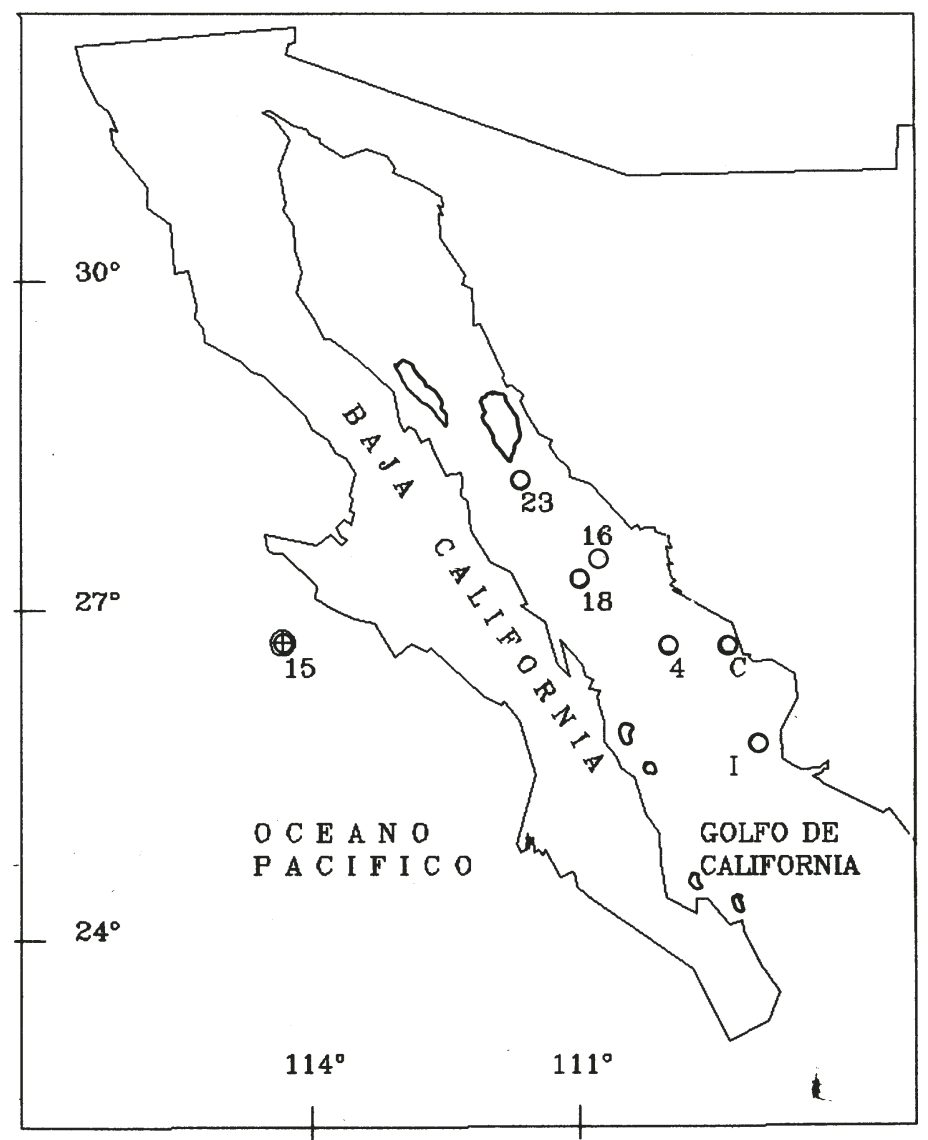

Fig. 1. Localización de los puntos de colecta de fitoplancton donde se encontraron Asteromphalus cleveanus (círculo cruzado) y A. flabellatus (círculos blancos). El número o letra junto a cada circulo es el de la estación. 
mente preparadas con Formvar. También se hicieron observaciones en microscopio electrónico de barrido (MEB) (Phillips 501, 10-12 kv), una vez que el material fue puesto y secado al aire en dispositivos de aluminio y posteriormente metalizado.

La terminología adoptada es la que se emplea comúnmente en estudios morfológicos de diatomeas (Anónimo, 1975; Ross et al., 1979). Terminología específica para Asteromphalus fue tomada de Gombos (1980) y Hernández-Becerril (1991), especialmente en referencia a estructuras como la porción central, líneas de separación, radios singular y ordinarios e indentación (Fig. 2).

Las preparaciones permamentes están depositadas en la colección particular del autor, y una de ellas será enviada a la colección de diatomeas del Instituto de Ciencias del Mar y Limnología, UNAM (MEXU-D), marcada XV (Asteromphalus cleveanus).

\section{OBSERVACIONES Y DISCUSION}

\section{Asteromphalus cleveanus Grunow Figs. 2, 3.}

Grunow In: Schmidt, 1876, pl. 38, figs. 13, 14; Rattray, 1889, p. 662; Allen y Cupp, 1935 , p. 123, fig. 23; Subrahmanyan, 1946, p. 106, figs. 84, 88; Wood, 1963, p.193, pl. 2 , fig. 28; Hendey, 1970, p. 114, pl. 4, fig. 42.

Non Asteromphalus cleveanus Grunow sensu Skvortzow, 1932, p. 267, pl. 4, fig. 1= Asteromphalus flabellatus (Bréb.) Grev.

=Asteromphalus wallichianus (Grev.) Ralfs sensu Cleve, 1873, p. 5, pl. 1, fig. 1.

Descripción en microscopio de luz. Célula de valvas elípticas, ligeramente convexas, en vista valvar. Porción central angulada, no redondeada, que se extiende de 1/3 a casi 1/2 del diámetro de la valva, con las líneas de separación rectas. Se encontraron ejemplares con 8 a 9 radios hialinos, el radio singular es más delgado y largo que los ordinarios; los radios ordinarios adyacentes al radio singular son más largos que los restantes. Cerca del radio singular se puede observar la presencia de la indentación, estructura encontrada en las especies de Asteromphalus, que en este caso es muy visible aún en el ML. El tipo de areolas de las porciones entre los radios es delicado, con 11 a 13 areolas/ $10 \mu \mathrm{m}$. Longitud de las valvas en el eje mayor: 39-41.5 $\mu \mathrm{m}$, anchura (longitud en el eje perpendicular al mayor): 28.5-30.5 $\mu \mathrm{m}$.

Relación con A. flabellatus. La especie A. cleveanus es realmente poco conocida y ha sido considerada como sinonimo de $A$. flabellatus por algunos autores (e.g. Sournia, 1968; Simonsen, 1974). Sin embargo, van Landinghan (1967) en su catálogo de nombres de diatomeas la cita como una especie válida.

Las principales diferencias entre las dos especies son: la forma de la valva, $A$. cleveanus es definitivamente elíptica, mientras que $A$. flabellatus varía de circular, subcircular a subelíptica (Figs. 4,5 ). También la forma y proporción relativa de la porción central es diagnóstica: en $A$. cleveanus es angulada y ocupa hasta casi $1 / 2$ del diámetro de la valva, en tanto que en $A$. flabellatus esta estructura es más redondeada, ocupando de 1/4 a 1/3 del diámetro valvar (fig. 5). 


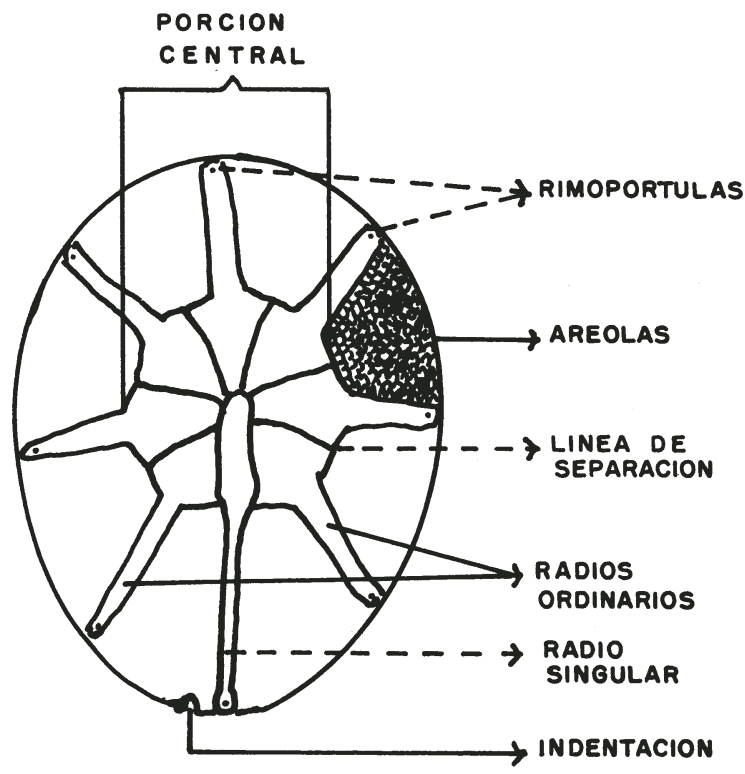

Fig. 2. Diagrama de Asteromphalus cleveanus, mostrando las principales estructuras de la especie.

El número de radios no parece ser tan importante en la separación de ambas especies, pues si bien aquí se encontraron ejemplares de $A$. cleveanus con 8 y 9 radios, Subrahamanyan (1946) y Hendey (1970) ilustraron ejemplares de 12 radios. La talla tampoco es decisiva en la separación especifica, ya que Allen y Cupp (1935) documentaron ejemplares de A. cleveanus de hasta $70 \mu \mathrm{m}$ en el eje mayor. En este estudio las valvas detectadas fueron en general mucho más pequeñas que las de $A$. flabellatus.

Ambas especies comparten caracteres como el tipo de areola (delicado, con 11-13 areolas $(10 \mu \mathrm{m}$ ) y el patrón de las líneas de separación (rectas, no en zig-zag). En $A$. flabellatus (como en todas las especies del género) se localizan procesos labiados (rimoportulae) en la parte más distal de cada radio hialino (Figs. 6, 7); la rimoportula del radio singular es más grande que las de los radios ordinarios (Fig. 6). Se asume que esta morfología básica está presente en $A$. cleveanus, basándose en las observaciones en ML.

Distribución de $A$. cleveanus. Esta especie no se ha registrado anteriormente en aguas de la corriente de California, siendo éste el primer registro en aguas costeras de Baja California. A. cleveanus fue encontrada solo en costas de Baja California $\left(26^{\circ} 32^{\prime} \mathrm{N}\right.$, $114^{\circ} 40^{\circ} \mathrm{W}$ ), mientras que A. flabellatus se detecto en muestras del Golfo de California (Fig. 1). No se encontraron ambas especies juntas en una muestra.

Por los datos obtenidos en la literatura, se puede decir que A. cleveanus es una especie de amplia distribución, aunque poco frecuente, en aguas tropicales y subtropicales de todo el mundo, pero especialmente en el Oceáno Indico. Se le debe considerar como especie nerítica y litoral, no oceánica. 

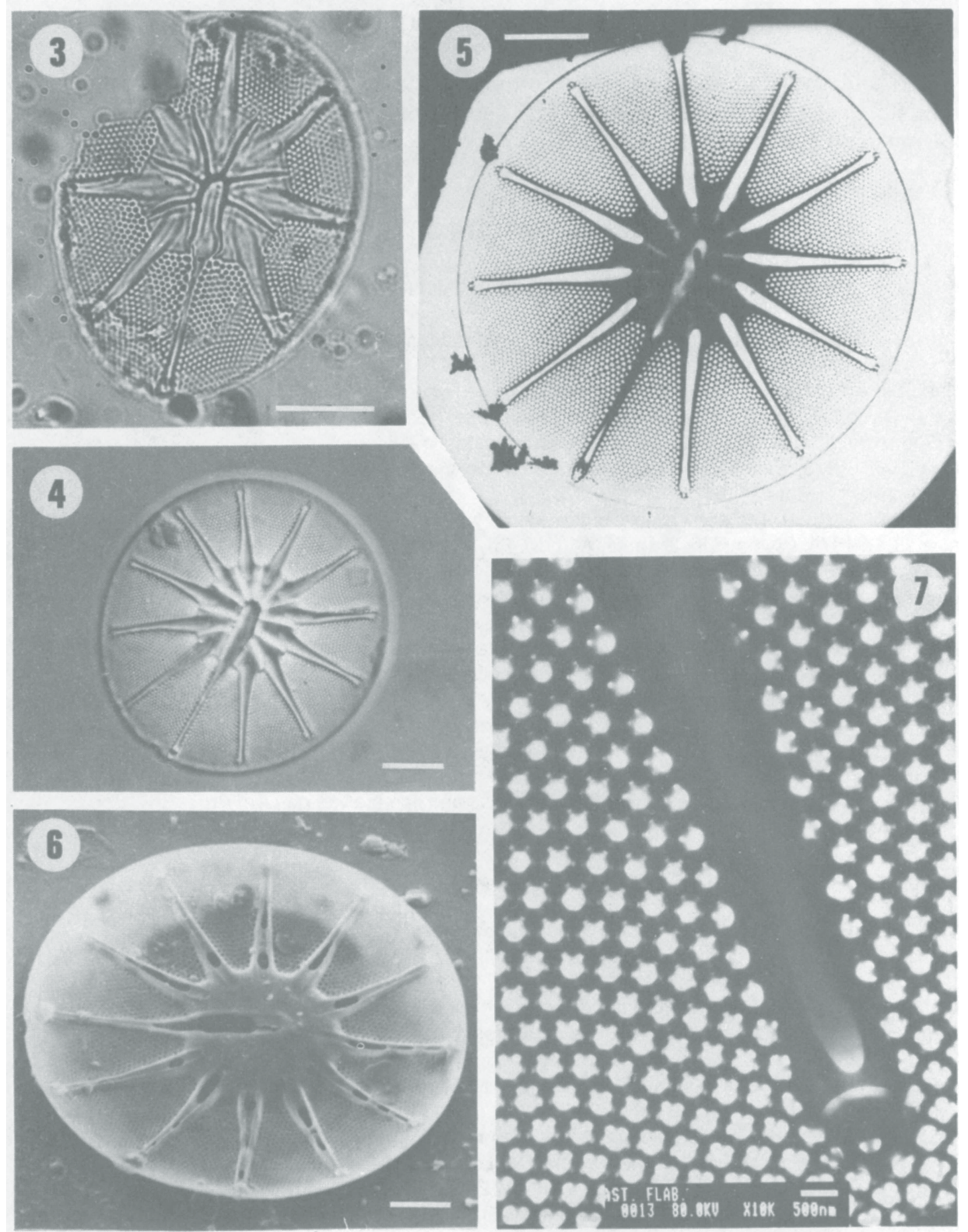

Figs. 3-7. Fig. 3 Asteromphalus cleveanus. Una valva típica, ML. Figs. 4-7. A. flabellatus. Fig. 4. Una valva completa, ML. Fig. 5. Otra valva completa. Nótense las rimoportulae al final de cada radio, MET. Fig. 6. Vista interna de una valva, MEB. Fig. 7. Detalle de un radio ordinario, al final del cual está una rimoportula, MET. Escala $=10 \mu \mathrm{m}$, Figs. $3-6 ;=0.5 \mu \mathrm{m}$, Fig. 7 


\section{AGRADECIMIENTOS}

Parte de este trabajo se realizó en el departamento de Botánica de la Universidad de Bristol, Inglaterra. El autor agradece la asesorla y los acertados comentarios del Dr. R. M. Crawford y del Prof. F. E. Round.

\section{LITERATURA CITADA}

Allen, W. E. \& E. E. Cupp. 1935. Plankton diatoms of the Java Sea. Ann. Jardin Bot. Buitenzorg 44: 101224.

Anónimo. 1975. Proposals for the standardization of diatom terminology and diagnosis. Nova Hedwigia, Beih. 53: 323-354.

Atilano Silva, H. M. 1987. Composición y estructura de la comunidad del fitoplancton silíceo en el Golfo de California, en marzo de 1983. Tesis Profesional. Facultad de Ciencias Marinas, Universidad Autónoma de Baja California. Ensenada, Baja California. $161 \mathrm{pp}$.

Cleve, P. T. 1873. Examination of diatoms found on the surface of the Sea of Java. Bih. K. Svenska Vetensk. Hand. 1 (11): 1-13.

Gombos, A. M. 1980. The early history of the diatom family Asterolampraceae. Bacillaria 3: 227-272.

Hasle, G. R. 1978. Diatoms. In: Sournia, A. (ed.). Phytoplankton manual. UNESCO. Paris, pp. 136-142.

Hendey, N. I. 1970. Some littoral diatoms of Kuwait. Nova Hedwigia, Beih. 31: 101-167.

Hernández-Becerril, D. U. 1988a. Planktonic dinoflagellates (except Ceratium and Protoperidinium) from the Gulf of California and of the coasts of Baja California. Bot. Mar. 31: 423-435.

Hernández-Becerril, D. U. 1988b. Observaciones de algunos dinoflagelados (Dinophyceae) del Pacifico Mexicano con microscopios fotónico y electrónico de barrido. Inv. Pesq. 52(4): 517-531.

Hernández-Becerril, D. U. 1989. The morphology and taxonomy of species of the diatom genera Asteromphalus and Chaetoceros. Ph. D. Thesis. Universidad de Bristol. Inglaterra. $138 \mathrm{pp}$.

Hernández-Becerril, D. U. 1991. The morphology and taxonomy of species of the diatom genus Asteromphalus Ehr. Bibliotheca Diatomologica 23, J. Cramer. 57 pp.

Rattray, J. 1889. Revision of the genus Coscinodiscus and some allied genera. Proc. Roy. Soc. Edinburgh 16: $449-692$

Ross, R., Cox, E. J., Karayeva, N. I., Mann, D. G., Paddock, T. B. B., Simonsen, R. \& Sims, P. A. 1979. An amended terminology for the siliceous components of the diatom cell. Nova Hedwigia, Beih. 64: 513-533.

Schmidt, A. 1876. Atlas der Diatomaceenkunde. Leipzig. lám. 38.

Simonsen, R. 1974. The diatom plankton of the Indian Ocean expedition of R. V. "Meteor" 1964-1965. Meteor. Forsch., Reih. D. 19: 1-66.

Skvortzow, B. K. 1932. Diatoms from the bottom of the Sea of Japan. Philippine J. Sci. 47(2): 265-280.

Sournia, A. 1968. Diatomées planctoniques du Canal de Mozambique et de l'ile Maurice. Mem. ORSTOM 31: $1-120$.

Subrahmanyan, R. 1946. A systematic account of the marine plankton diatoms of the Madras coast. Proc. Indian Acad. Sci., sect. B 24(4): 85-197.

Van Landingham, S. L. 1967. Cataloque of fossil and recent genera and species and their synonyms. Part I. Acanthoceras through Bacillaria J. Cramer, Lehre. pp. 1-493.

Wood, E. J. F. 1963. Studies on Australian and New Zealand diatoms. VI. Tropical and subtropical species. Trans. Roy. Soc. New Zealand, Botany 2(15): 189-218. 